\title{
De l'argument à l'action : la biodiversité au service des jardins familiaux
}

From the Argument to Action: Biodiversity at the Service of Allotment Gardens

Jean Noël Consalès, Sophie Joimel, France Cordier, Cloé Jareno, ÉlodieDenise Chenot et Christophe Schwartz

\section{(2) OpenEdition}

\section{Journals}

Édition électronique

URL : http://journals.openedition.org/paysage/9603

DOI : $10.4000 /$ paysage.9603

ISSN : 1969-6124

\section{Éditeur :}

École nationale supérieure du paysage de Versailles-Marseille, Institut national des sciences appliquées Centre Val de Loire - École de la nature et du paysage, École nationale supérieure d'architecture et de paysage de Bordeaux, École nationale supérieure d'architecture et de paysage de Lille, Agrocampus Angers

Référence électronique

Jean Noël Consalès, Sophie Joimel, France Cordier, Cloé Jareno, Élodie-Denise Chenot et Christophe Schwartz, « De l'argument à l'action : la biodiversité au service des jardins familiaux », Projets de paysage [En ligne], 13 | 2015, mis en ligne le 31 décembre 2015, consulté le 04 septembre 2020. URL http://journals.openedition.org/paysage/9603 ; DOI : https://doi.org/10.4000/paysage.9603

Ce document a été généré automatiquement le 4 septembre 2020.

Projets de paysage 


\section{De l'argument à l'action : la biodiversité au service des jardins familiaux}

From the Argument to Action: Biodiversity at the Service of Allotment Gardens

Jean Noël Consalès, Sophie Joimel, France Cordier, Cloé Jareno, ÉlodieDenise Chenot et Christophe Schwartz

$1 \mathrm{Au}$ cours de leur longue histoire, les jardins familiaux ${ }^{1}$ ont connu des phases d'évolution très marquées. En plein essor durant les périodes de crise $\mathrm{du} \mathrm{xx}^{\mathrm{e}}$ siècle, notamment pendant les deux guerres mondiales, ils ont enregistré un fort déclin durant les Trente Glorieuses (Cabedoce et Pierson, 1996). Depuis peu, ils jouissent d'un vif regain d'intérêt et participent pleinement des réflexions plus générales sur l'agriculture urbaine (Aubry, 2014). À cet égard, la recherche française les analyse le plus souvent à travers le prisme de la multifonctionnalité (Fleury et Donadieu, 1997 ; Consalès, 2000; Scheromm, 2013), faisant ainsi écho à l'abondante littérature scientifique anglo-saxonne et notamment nord-américaine qui leur est consacrée (Duchemin et al., 2010 ; Wegmuller et Duchemin, 2010 ; Lovell, 2010). Leurs fonctions sociales restent néanmoins les mieux renseignées (Guyon, 2008). À la suite de travaux précurseurs (Dubost, 2000 ; Weber, 2000), de nombreuses analyses démontrent la large gamme de représentations, d'attributions, de pratiques et d'usages qu'ils suscitent, et ce, dans des contextes urbains très différents. Jusqu'alors plus estimées que quantifiées, leurs fonctions économiques, productives et alimentaires donnent lieu à de récentes investigations tentant de mesurer précisément leur potentiel nourricier (Pourias et al., 2014). Il en va de même pour leurs fonctions environnementales qui, en dehors de quelques travaux précoces (Schwartz, 1993), n'avaient, jusqu'à peu (projet ADEMESOJA), jamais suscité d'études réellement fouillées. Par conséquent, si les jardins familiaux restent encore des "terres inconnues" (Chenot et al., 2012) aux portées environnementales incertaines, ils suscitent de plus en plus de recherches émanant des sciences du vivant qui poussent à dépasser le cadre théorique de la multifonctionnalité pour tendre vers le concept, mieux adapté (Bonnal et al., 2012), de «services 
écosystémiques » (Millennium Ecosystem Assessment, 2005). En tant qu'espaces urbains structurés par la végétation, ils interrogent, notamment, quant à leur capacité à maintenir ou à générer de la biodiversité.

Mais, force est de constater que la tendance nationale en faveur des jardins familiaux que traduit cet intérêt scientifique grandissant ne masque pas, à l'échelle locale, d'énormes disparités de situations révélant une certaine ambiguïté territoriale à l'égard de ce type de potagers. Bien souvent, les maîtrises d'ouvrage leur préfèrent, en effet, des formes beaucoup moins contraignantes, au premier rang desquelles figurent les très appréciés jardins partagés (Frauenfelder, 2014). Dans des cas extrêmes, les jardins familiaux sont perçus comme de véritables réserves foncières en attente d'urbanisation. Face à cet état de fait, leurs structures gestionnaires associatives sont de plus en plus appelées à déployer des stratégies de promotion, de valorisation, de défense et de projet au sein desquelles la biodiversité est pleinement mobilisée. Quels sont donc les tenants et les aboutissants de cette mobilisation? Quelles acceptions ces structures gestionnaires donnent-elles à la biodiversité? Quelle est la portée écologique réelle des jardins familiaux? La biodiversité convoquée est-elle force de projet au sein de ces groupes de potagers dont les formes et les aménagements n'ont guère changé depuis la fin du xix siècle? Autant de questions auxquelles nous nous proposons de répondre dans le présent article en conjuguant une approche projectuelle, destinée à évaluer la capacité d'entraînement qu'exerce la notion de biodiversité sur les acteurs des jardins familiaux, et une approche environnementale dédiée à la mesure de la biodiversité réelle de ces potagers. Inscrit dans le cadre du programme ANR JASSUR (ANR-12-VBDU-0011, Jardins associatifs urbains), notre propos confronte, pour ce faire, deux échelles d'analyse :

- l'échelle nationale, en considérant la manière dont la Fédération nationale des jardins familiaux et collectifs utilise la biodiversité comme élément fédératif (à partir de l'étude du contenu de sa revue Jardin familial de France) et comme argument projectuel (notamment à partir de l'analyse du discours de son bureau d'études) ;

- l'échelle locale, en décrivant la manière dont certaines associations et leurs jardiniers mettent la biodiversité (démontrée grâce à des analyses écologiques précises) au service de leurs projets de maintien et de développement de sites de jardins familiaux sis dans des contextes fonciers particulièrement tendus (une étude de cas portant sur Marseille sera ici mobilisée).

\section{À l'échelle nationale : la biodiversité comme stratégie}

\section{La biodiversité en question}

Créée en 1896 par l'abbé Lemire, la Ligue française du coin de terre et du foyer est rebaptisée Fédération nationale des jardins familiaux et collectifs (FNJFC) en 2006. Forte de sa longue expérience et de son titre d'utilité publique (acquis en 1909), cette structure fédérative fait office de référence nationale en matière de jardins familiaux. Présente sur la quasi-totalité du territoire, elle agit à deux niveaux. Au niveau national, elle regroupe 250 structures associatives et assume la promotion du mouvement, notamment auprès des collectivités territoriales. En outre, elle gère soit en direct (en région parisienne) soit par l'intermédiaire de ses comités locaux, 85 groupes de potagers, notamment sur les terrains dont elle est propriétaire. Elle assure également 
la défense des associations et œuvre en faveur de l'avancement de la législation. Au niveau local, elle tient le rôle de conseiller juridique et technique des associations membres et organise la liaison entre les institutions de gestion. Pour ce faire, elle dispose de deux outils essentiels :

- un journal, intitulé Jardin familial de France, diffusé auprès des adhérents des associations affiliées;

- un bureau d'études, Sites et Jardins, spécialisé dans l'accompagnement de projets et la création de jardins familiaux.

Bien qu'encore centrale dans la sphère des jardins collectifs, la place de la FNJFC doit être relativisée. En effet, depuis le milieu des années 1990, la diversification des formes de potagers urbains entraine une multiplication des acteurs et des réseaux nationaux qui, faute de gouvernance globale, génère une concurrence larvée au sein d'un secteur pourtant associatif. L'inscription de plus en plus locale des éléments impactant directement sur les groupes de jardins familiaux (décisions administratives, règlements d'urbanisme, projets d'aménagement, etc.) joue, par ailleurs, en défaveur d'une organisation fédérative descendante datant du XIX $x^{e}$ siècle et explique de nombreuses défections. Enfin, la FNJFC doit encore composer avec certaines pratiques locales héritées du passé (clubbisation de l'espace, entre-soi, modes de culture peu respectueux de l'environnement, etc.) qui desservent son image. Face à cette situation, les instances fédératives qui se succèdent à la tête de la FNJFC depuis les années 2000 génèrent un puissant processus de modernisation fondé sur les principes du développement durable (Consalès, 2008). La logique est simple : la promotion de la fédération passe par la réaffirmation de l'utilité des jardins familiaux. À cet égard, il s'agit notamment de démontrer qu'en tant que lieux de nature urbaine, ces derniers sont support d'une biodiversité qui fournit des services écosystémiques variés aux territoires. Ce faisant, la biodiversité revêt une valeur programmatique importante rendant, en retour, service aux jardins familiaux. Cette logique induit un positionnement renouvelé de l'ensemble des acteurs fédératifs (instances dirigeantes, associations et jardiniers affiliés) sur les questions environnementales et se construit au rythme de l'évolution de considérations plus globales.

5 Nous avons donc cherché à comprendre à partir de quel moment et de quelle manière les concepts et les notions en lien avec la biodiversité ont été mobilisés et intégrés par la FNJFC. Il nous a semblé extrêmement difficile d'instruire ces questions par des méthodes traditionnelles d'investigation (entretiens). Nos enquêtes, réalisées sous forme d'entretiens auprès de 5 personnes ressources, nous ont en effet démontré que l'écologisation des considérations et des pratiques est difficilement conscientisée par les acteurs fédératifs, notamment dans sa dimension temporelle. Afin de pallier le manque d'informations précises, nous nous sommes tournés vers les discours textuels formulés, par la FNJFC, dans son magazine Jardin familial de France. Nous avons constitué un corpus de 56 numéros disponibles sous format numérique ${ }^{2}$, soit 10 années de publication d'un magazine bimestriel ${ }^{3}$. La numérisation du corpus et l'organisation chronologique des données nous ont permis, dans un premier temps, d'appliquer à la série un traitement quantitatif simple de lexicométrie réalisé via le logiciel Tropes ${ }^{4}$. Nous avons alors interrogé une liste de mots indicateurs ${ }^{5}$ et étudié leur répartition chronologique dans l'ensemble du corpus. En fonction de cette répartition, nous sommes revenus plus finement au sein du corpus pour, dans un second temps, analyser de manière qualitative l'évolution du sens donné à chacun des mots indicateurs durant 
les grandes périodes et les grands moments identifiés par le logiciel. Pour préciser notre propos, nous avons utilisé les informations, acquises lors des entretiens réalisés auprès des 5 personnes ressources de la FNJFC.

Figure 1. Évolution de l'occurrence des mots « biodiversité ", " écologie » et " mauvaises herbes » dans la revue Jardin familial de France de 2006 à 2015

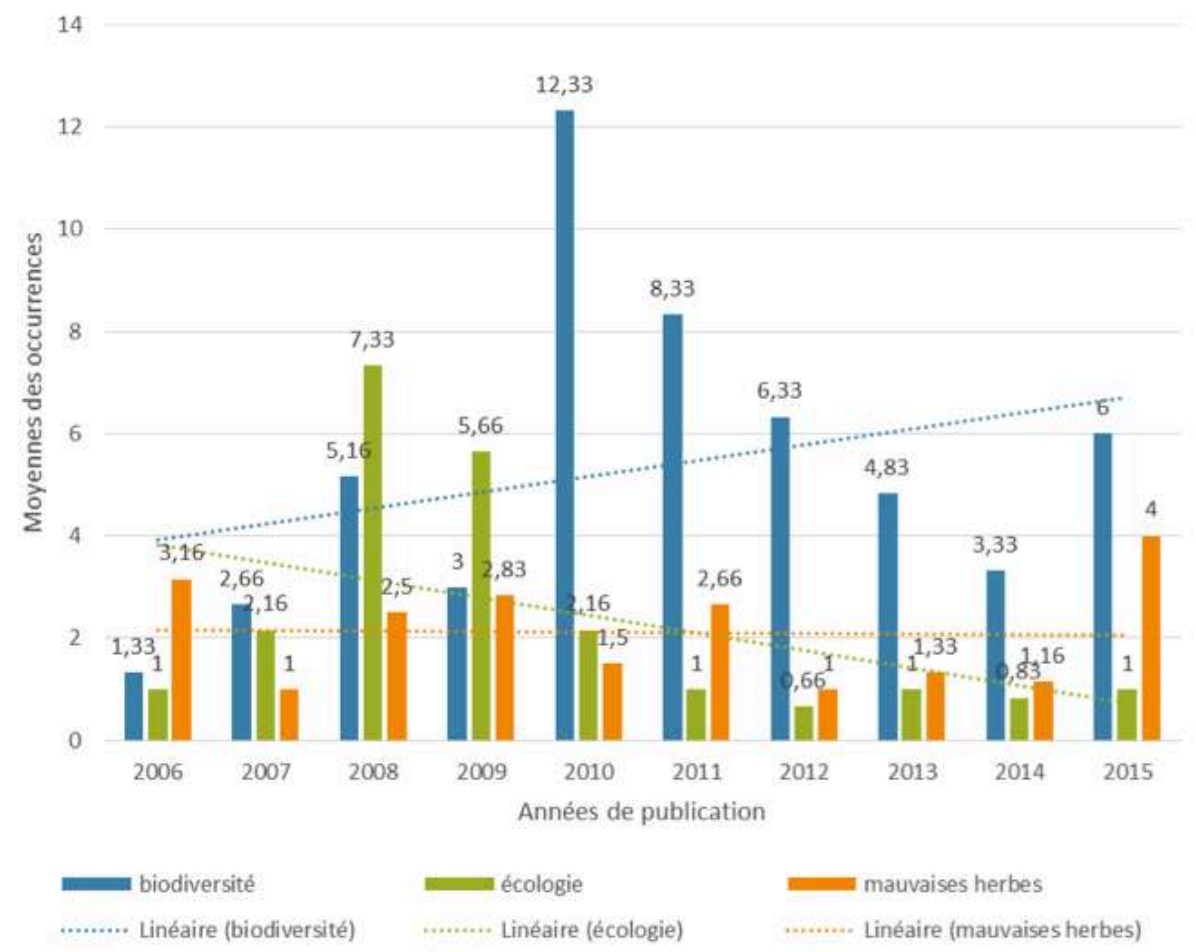

Elle est exprimée par la moyenne des occurrences pour chaque année de publication.

6 La figure ci-dessus présente les résultats les plus saillants de notre démarche. Elle montre, en effet, que la croissance continue de l'utilisation du terme "biodiversité » dans la revue Jardin familial de France est liée à deux événements d'envergure : l'un de portée nationale avec l'adoption par l'assemblée nationale du premier Grenelle de l'environnement en 2008 et l'autre de portée internationale avec l'année internationale de la biodiversité en 2010. Dans son éditorial du numéro 460 de mai/juin 2010, le président de l'époque précise à ce propos :

«L'ONU a déclaré 2010 année internationale de la biodiversité [...], nous devons donc préserver cette biodiversité et si possible encore l'enrichir [...]. En nous inscrivant dans cette démarche respectueuse de l'environnement, nous contribuons aussi à pérenniser nos jardins et à les développer. Les jardins familiaux collectifs sont les maillons indispensables des "trames vertes" que les collectivités locales vont devoir créer dans toutes les villes pour permettre le déplacement et la migration des espèces vivantes. "

7 Depuis, la biodiversité fait partie intégrante du vocabulaire usuel de la FNJFC. Pour la qualifier, cette dernière utilise la définition donnée par la convention sur la biodiversité écologique adoptée à Rio, en 1992 :

«La biodiversité ou diversité biologique désigne la variabilité des organismes vivants de toute origine, y compris, entre autres, les écosystèmes terrestres, marins et autres écosystèmes aquatiques et les complexes écologiques dont ils font partie. 
Cela comprend la diversité au sein des espèces et entre espèces ainsi que celle des écosystèmes " (Jardin familial de France, $\mathrm{n}^{\circ} 459$ ). pratiques :

«Nous focalisons souvent notre attention sur les animaux dits "nuisibles" et les exterminons systématiquement, sans imaginer que leurs prédateurs naturels sont, eux aussi, potentiellement présents et dépendent de ces mêmes "nuisibles". Il en va de même pour les plantes sauvages communément appelées "mauvaises herbes", pour lesquelles nous n'avons aucune considération alors qu'elles sont la principale source de nourriture pollen et nectar des insectes pollinisateurs qui, à leur tour, féconderont les fleurs de nos cultures " (ibid., $n^{\circ} 487$ ).

11 Loin d'être un simple élément consensuel de langage ou de communication, la biodiversité devient, dans le même temps, une véritable piste d'actions concrètes destinée à asseoir la stratégie de la FNJFC en matière de développement durable et d'environnement. À partir de 2013, cette tendance conduit la Fédération à enclencher un volet opérationnel et projectuel. Celui-ci sous-tend des modifications au sein du bureau d'études qui lui est associé.

\section{La biodiversité en projet}

12 Créé en 1994, le bureau d'études associé à la FNJFC est devenu Sites et Jardins en 2013. Spécialisé dans la conception de jardins familiaux, il propose une méthode de création en quatre phases ${ }^{6}$ pouvant s'apparenter à un projet de paysage (Corajoud, 2000). Jusqu'à récemment, ses missions de diagnostic, de programmation ou de maîtrise d'œuvre étaient assurées par une petite équipe essentiellement composée de paysagistesconcepteurs. Cependant, la montée en puissance des considérations et des pratiques fédératives en matière d'environnement, en général, et de biodiversité, en particulier, a conduit à l'affirmation d'autres compétences salariales. De fait, l'équipe est aujourd'hui principalement constituée d'environnementalistes et le bureau d'études semble guidé par des savoirs et savoir-faire bien plus naturalistes qu'urbanistiques et projectuels. 
Lorsqu'un projet les réclame, ces dernières compétences sont agrégées par association ou groupement avec des architectes, des sociologues et des urbanistes.

Cette évolution de l'approche du bureau d'études, justifiée par l'apparent renouvellement de la commande et des exigences des maîtrises d'ouvrage, sous-tend alors des partis pris d'aménagement fondés sur les principes du développement durable. À ce propos, le directeur de Sites et Jardins explique :

«Dans les faits quand on faisait des plans, on avait conscience qu'il ne fallait pas que la création de jardins familiaux impacte l'environnement. On est parti sur le principe de départ qu'il fallait que cela laisse le moins de traces possible, si jamais un jour ça devait disparaître. [...] quand on part il ne faut pas qu'on laisse des fondations énormes, des choses non recyclables, etc. » (entretien, juillet 2015).

Les projets de création de jardins familiaux donnent donc lieu à des aménagements favorables à la biodiversité, relevant parfois de la petite ingénierie écologique. Il s'agit, par exemple, d'opter pour des clôtures à maille large permettant le déplacement de la faune ou de planter, dans les espaces collectifs, des prairies naturelles plutôt que des gazons afin de permettre aux insectes de s'installer et à la flore de s'exprimer. Dans le même ordre d'idée, les petits fruits (framboises, cassis, groseilles) sont utilisés dans les parties communes pour satisfaire aussi bien les jardiniers que les animaux. Parfois, des vergers de variétés anciennes sont introduits. Ce souci de la biodiversité s'exprime également au sein des plans de gestion des sites créés ou des règlements intérieurs établis par le bureau d'études. Intégrant les préceptes de la gestion différenciée, ces documents préconisent, au-delà des espaces cultivés, le maintien de zones de fauches tardives ou d'entretien minimal :

«Si vous voulez avant le règlement, en hiver, c'était de retourner le terrain à grosse motte pour que le jardin soit propre. Cette notion de propreté, c'est juste incroyable! On lutte, depuis 2008, mais ces dernières années encore plus pour changer nos règlements intérieurs en disant: ne mettez plus vos jardins propres l'hiver! Laissez les plantes sauvages fanées qui sont des abris par excellence à une faune sous forme de larves!» (entretien avec le directeur de Sites et Jardins, juillet 2015).

15 Par ailleurs, le port naturel est recommandé pour la taille des végétaux ainsi que le fleurissement des parties non productives (allées, abords de cabanes, etc.). Le "zéro pesticides » est également fortement prescrit. Enfin, au travers des plans de gestion ou des règlements intérieurs proposés, le bureau d'études travaille aussi sur la biodiversité cultivée :

«On insiste sur la rotation et la variété des cultures [...] c'est de la biodiversité même si c'est de la biodiversité horticole. Elle passe par la culture de différentes variétés de légumes qui réduisent aussi le risque de maladies" (entretien avec le directeur de Sites et Jardins, juillet 2015).

16 Relayées par Jardin familial de France, ces initiatives sont souvent réinterprétées et adaptées dans les jardins familiaux existants, à l'initiative d'associations de plus en plus éclairées sur la biodiversité. Dans l'immense majorité des cas, cette dynamique se traduit par des microaménagements pour lesquels Site et Jardins est parfois consulté : installation de mares, de ruchers (en collaboration avec des apiculteurs locaux), de nichoirs, d'hôtels à insectes. À l'échelle locale s'inventent ainsi des bonnes pratiques que réceptionne, traduit et retransmet la FNJFC, notamment par l'intermédiaire de son magazine. La biodiversité contribue, par conséquent, à établir des rapports ascendants dans l'organisation verticale de la Fédération et à susciter une gouvernance renouvelée. 


\section{À l'échelle locale : la biodiversité comme argument}

L'échelle locale semble donc de plus en plus pertinente pour analyser de manière fine l'émergence de la biodiversité au sein des jardins familiaux. Nous avons souhaité mettre à l'épreuve de terrains marseillais cette assertion. Sur la seule commune de Marseille toutes les formes de jardins collectifs sont, en effet, présentes et les quelques 1000 parcelles de jardins familiaux dépendent d'associations soit non affiliées, soit affiliées, soit directement intégrées (comité local) à la FNJFC. Cette diversité de situations et de statuts, quasi unique en France, justifie l'étude de cas.

\section{La biodiversité pratiquée}

Afin d'évaluer les considérations et les pratiques des jardiniers marseillais en matière de biodiversité, nous avons effectué une enquête auprès de 36 individus, dans dix sites de jardins familiaux différents ${ }^{7}$. Celle-ci a été réalisée, in situ, sous la forme d'un questionnaire à passation directe dans lequel certaines questions d'opinion sont restées ouvertes. Lors de la préenquête, le terme de biodiversité est apparu comme trop peu maîtrisé par la population sondée pour être instruit directement. Il a donc été remplacé par le terme plus générique d'environnement. Plus qu'à l'écologie, celui-ci reste associé, par les jardiniers enquêtés, au cadre de vie. De fait, il revêt une dimension spatiale forte. En effet, ce terme d'« environnement » est avant tout défini en tant que lieu de détente (pour $21 \%$ des jardiniers), de nature (19\%), en dehors de la pollution et de la ville ( $12 \%$ ) ou de convivialité (11\%). Il est également synonyme de santé physique et mentale ( $11 \%)$. Il relève, par ailleurs, d'un intérêt particulier pour les jardiniers marseillais. Ainsi, lorsqu'il leur est demandé de jauger l'importance qu'ils attribuent à la notion d'environnement sur une échelle de valeur allant de 1 à 10 , la plupart d'entre eux attribuent une note située entre 7 à 10.

Figure 2. Notes attribuées à la notion d'environnement par les jardiniers marseillais

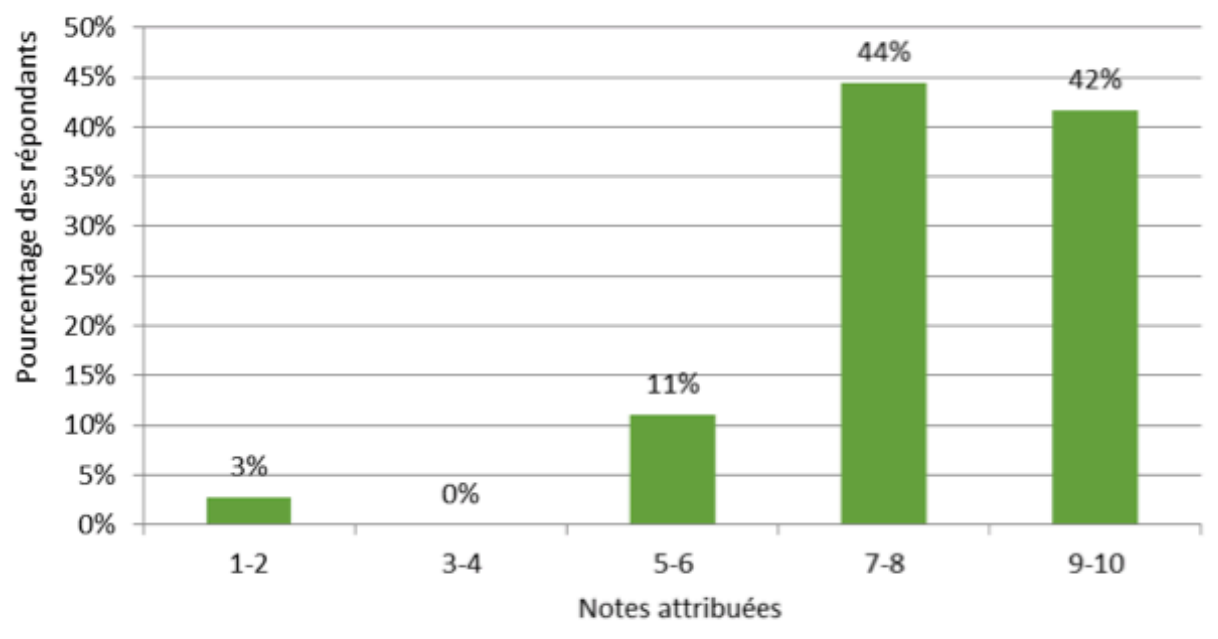

$19 \mathrm{Au}$ jardin, cet intérêt se traduit essentiellement par des pratiques déclarées comme étant plus respectueuses et déterminées par rapport à un modèle d'agriculture biologique aussi idéalisé que méconnu. À ce titre, $75 \%$ des jardiniers marseillais 
estiment avoir des pratiques proches de l'agriculture biologique. Selon les individus enquêtés, cette dernière se définit par l'absence de produits chimiques $(41 \%)$ ou par leur utilisation raisonnée $(28 \%)$ ainsi que par des pratiques qualifiées de "naturelles " (18\%). À l'inverse, elle relève pour d'autres (8\%) de la «croyance » ou de l'effet de mode. Dans les faits, la moitié des sondés utilisent des produits phytosanitaires, parfois réduits aux seuls anti-limaces. L'amendement et l'utilisation d'engrais sont des pratiques très courantes (respectivement $92 \%$ et $55 \%$ ) mais se fondent de plus en plus sur des produits non chimiques. Le développement du compostage, effectué par $67 \%$ des enquêtés, témoigne de cet état de fait. Facteur majeur de modification de la biodiversité des jardins, le travail du sol est réalisé par $100 \%$ des sondés (motoculteur, bêchage, binage, griffage, ratissage). $72 \%$ des jardiniers marseillais utilisent le motoculteur, technique parmi les plus destructurantes pour les sols. $60 \%$ d'entre eux le passent jusqu'à quatre fois dans l'année. Le bêchage est également très courant (89\%) et souvent mensuel (75\%). Quoi qu'il en soit, sans être réellement en mesure de la nommer ou de la définir, les jardiniers marseillais agissent en faveur d'une biodiversité qu'ils ne rationalisent certes pas en tant que telle mais qu'ils incarnent fortement, désignant oiseaux, animaux, insectes, végétaux, plantes et arbres ou développant parfois d'étonnants savoirs et intérêts naturalistes. Ainsi, $61 \%$ des sondés déclarent avoir installé, sur leur parcelle, de petits équipements pour la faune : nichoirs ou réserves de nourriture (boules de graisse, tournesols) pour les oiseaux (57\%), hôtels à insectes $(11 \%)$. En matière de biodiversité végétale, les personnes enquêtées ne semblent pas conscientiser leur impact sur la flore spontanée. En revanche, la biodiversité cultivée suscite un vif intérêt de jardiniers toujours en quête de légumes soit plus savoureux (recherche de variétés anciennes notamment), soit mieux adaptés au climat méditerranéen (incorporation d'espèces associées à d'autres terroirs comme récemment la cristophine). Derrière l'apparente uniformité des cultures (en automnehiver, $75 \%$ des jardiniers cultivent des choux, $70 \%$ des salades ; au printemps-été, $98 \%$ cultivent des tomates, $94 \%$ des courges, $92 \%$ des haricots verts, $87 \%$ des radis, $83 \%$ des aubergines, $75 \%$ des fraises) se cache, en réalité, une grande diversité d'espèces et de variétés.

Figure 3. Nombre d'espèces cultivées mentionné par les jardiniers marseillais

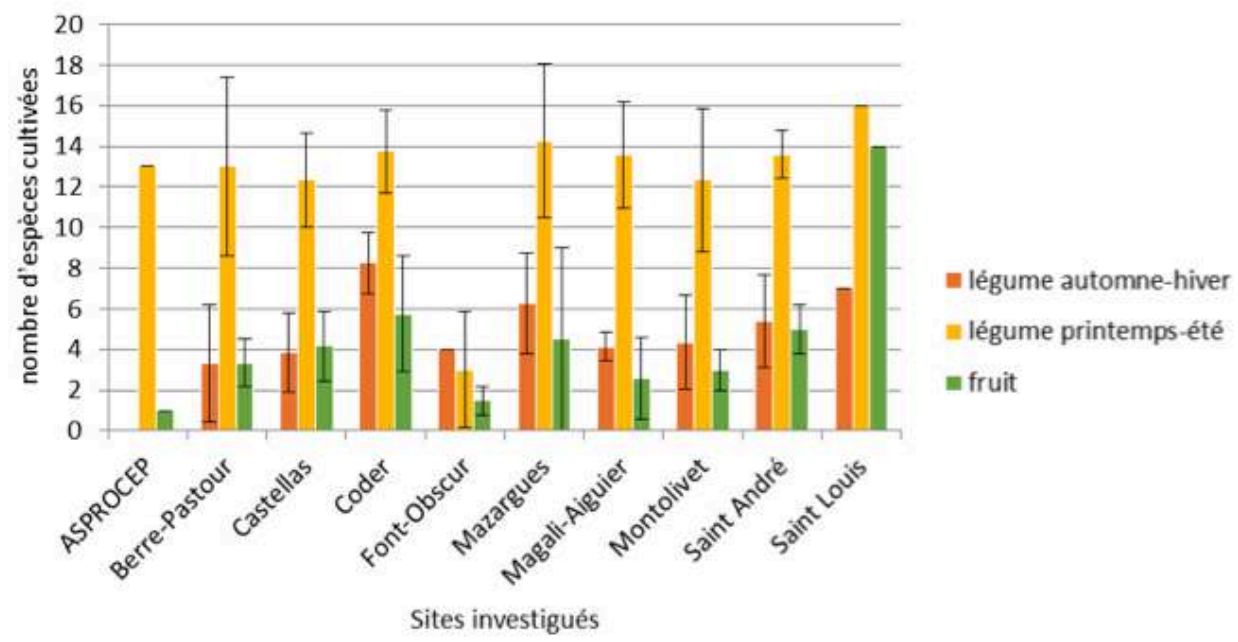


vers des modes de penser et de faire identifiés comme favorables à la biodiversité L'utilisation des intrants de synthèse, parfois à hautes doses, ainsi que l'intensité du travail du sol restent cependant des enjeux majeurs en la matière. Par ailleurs, la flore spontanée et la faune du sol demeurent des impensés de l'écosystème du potager. Pourtant, dans les jardins familiaux, leurs valeurs écologiques sont loin d'être négligeables.

\section{La biodiversité mesurée}

En nous fondant sur les pratiques des 36 jardiniers marseillais interrogés et sur les caractéristiques physico-chimiques des terres de surface de leurs parcelles, nous avons distingué 5 jardins statistiquement représentatifs pour effectuer des relevés floristiques et faunistiques, au printemps et à l'automne 2013. Afin d'étudier la biodiversité taxonomique de ces jardins familiaux, nous avons choisi de centrer nos recherches sur trois groupes distincts sélectionnés :

- la flore cultivée, pour mesurer l'incidence des jardiniers et de leur potager sur la biodiversité agronomique ;

- la flore spontanée, car elle influence directement ou indirectement les processus écosystémiques en modifiant la quantité et la qualité des habitats et des substrats utilisés par d'autres organismes (Berendse, 1998 ; Díaz et Cabido, 2001 ; Hooper et al., 2005) ;

- la mésofaune des sols (déterminée grâce à la méthode des quadrats ${ }^{8}$ ), pour son rôle de bioindicateur de la qualité des sols et de leur pollution (Cortet et al., 1999; Santorufo et al., 2012), composée majoritairement des microarthropodes (collemboles et acariens).

Figure 4. Diversité taxonomique (richesse spécifique) détectée par l'inventaire complet de la flore spontanée et cultivée et par la méthode des quadrats pour les collemboles des jardins au sein de cinq parcelles de jardins familiaux marseillais

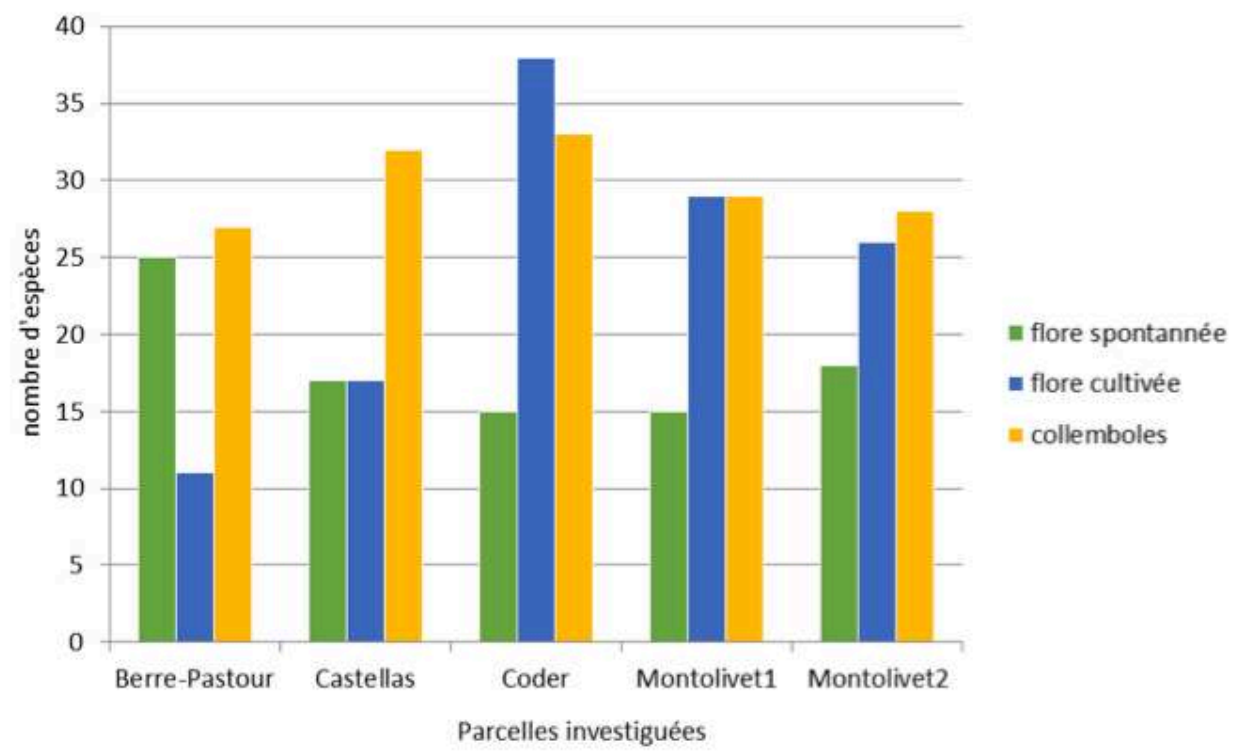

Comme nous l'avons vu précédemment, la biodiversité cultivée des jardins familiaux marseillais est élevée tant en termes d'espèces plantées (voir figures ci-dessus et ci- 
dessous) que de variétés mobilisées. En effet, au sein des cinq jardins familiaux investigués, les jardiniers déclarent cultiver un total de 67 espèces différentes, essentiellement légumières, estivales et méditerranéennes. Bien que dominante, la vocation productive et potagère de ces espaces n'exclut pas la culture de nombreuses espèces de fleurs et d'arbres. En revanche, peu de surfaces sont consacrées aux pelouses et à la friche. Cet état de fait n'empêche pourtant pas la présence d'un total de 41 espèces végétales spontanées appartenant à 20 familles distinctes. Cinq de ces espèces sont présentes dans tous les jardins: taraxacum officinale F.H. Wigg 1780 (pissenlit commun), poa annua L. 1753 (pâturin annuel), stellaria media (L.) Vill 1789 (stellaire), Veronica persica Poir. 1808 (véronique) et trifolium repens L. 1753 (trèfle blanc). Leur présence est aussi commune que dans d'autres habitats urbains tels que les jardins privés (Smith et al., 2006), les pelouses ou les pieds d'arbres. Elle s'explique par la propension élevée de ces plantes à la dispersion et à leur grande capacité d'installation en milieu urbain (Aronson et al., 2007). Les jardins familiaux semblent donc peu propices à l'établissement d'espèces soit pérennes (les plantes annuelles dominent), soit plus exigeantes en quantité de substrat disponible. Le profil de leur communauté végétale correspond à celui fréquemment retrouvé dans la flore herbacée du milieu urbain (Kleyer, 1999 ; Knapp et al., 2012).

Par ailleurs, dans les cinq jardins familiaux investis, 55 espèces de collemboles ont été identifiées, avec une moyenne de 30 espèces par jardin (voir figure ci-dessus). La diversité taxonomique et l'abondance ici observées sont supérieures à celles mesurées en milieu agricole, notamment pour les grandes cultures (Joimel et al., 2014). Les collemboles présents dans ces potagers sont des taxons à large répartition (mesaphorura sp., proisotoma minuta Tullberg 1871, protaphorura armata Tullberg 1869), repérés dans de nombreuses études, y compris en milieux urbains (Kuznetsova, 2003; Santorufo et al., 2014). En revanche, le fait que $P$. minuta et $P$. armata soient parmi les espèces les plus recensées est peu courant dans la littérature. En milieu tempéré, l'espèce paristoma notabilis Schäffer 1896 domine généralement (Potapow, 2001). L'abondance de folsomia similis Bagnall 1939 est, elle aussi, peu commune dans les études sur les microarthropodes. Il convient enfin de souligner la présence d'espèces associées au milieu méditerranéen telles que proctostephanus stukeni Börner, C, 1902.

$24 \mathrm{Au}$ final, les jardins familiaux marseillais investigués apparaissent comme des réservoirs d'une biodiversité certes ordinaire (Mougenot, 2003) mais abondante, au sein desquels la faune du sol semble particulièrement remarquable. Peu visible et encore méconnue, ce n'est pourtant pas cette biodiversité qui est mobilisée par les associations pour défendre les sites de potagers menacés par l'urbanisation.

\section{La biodiversité mobilisée}

À Marseille, le statut foncier des jardins familiaux est ambigu (Consalès, 2000). Si certains sites se créent ou sont en cours de création, d'autres sont directement menacés par l'urbanisation. Dans ce dernier cas, les structures de gestion doivent en permanence trouver des arguments pour légitimer l'existence des potagers, face à des besoins jugés prioritaires: logements, activités, déplacements, etc. En la matière, l'association des Jardins ouvriers et familiaux de Provence (non affiliée à la FNJFC) fait office de précurseur, sur son jardin du Castellas. Avec ses 246 parcelles et ses 14 hectares de terrain, ce site de jardins familiaux est le plus important de Marseille. Dans les documents d'urbanisme locaux, il est pourtant affecté, dans une partie, à 
l'urbanisation et fait l'objet, dans une autre partie, d'un classement de réservation pour le développement de la voirie (projet de rocade L2). Conscient de son indéniable attrait paysager, le bureau de l'association œuvre, depuis plusieurs années, pour que le site soit reconnu en tant qu'élément majeur du patrimoine naturel de Marseille. Pour ce faire, il se place dans une stratégie d'ouverture et de communication fondée sur la valorisation de la faune et de la flore. À l'intérieur du jardin, un sentier permet ainsi aux visiteurs de découvrir les richesses du lieu, notamment en matière de biodiversité sauvage et cultivée. Un ancien dirigeant local de la FNJFC affirme à ce sujet :

«Face à la menace de l'urbanisation, la tactique de l'association du Castellas est la meilleure. Il faut accroître la lisibilité des sites. Au quotidien, il faut les ouvrir, ouvrir, ouvrir et encore ouvrir. Les blogs et les pétitions ne suffisent pas, car la plupart des riverains ne savent même pas que les jardins existent. Il faut progressivement transformer les jardins en véritable parc de proximité, afin que les habitants du quartier s'en saisissent réellement. Pour faire cela, il faut que les jardiniers comprennent que la somme de leurs intérêts individuels ne fait pas l'intérêt public et que contre une logique d'utilité publique il faut opposer une autre logique d'utilité publique» (entretien avec un ancien dirigeant de la FNJFC, décembre 2012).

Figure 5. Les jardins familiaux de Mazargues, biodiversité contre urbanisation

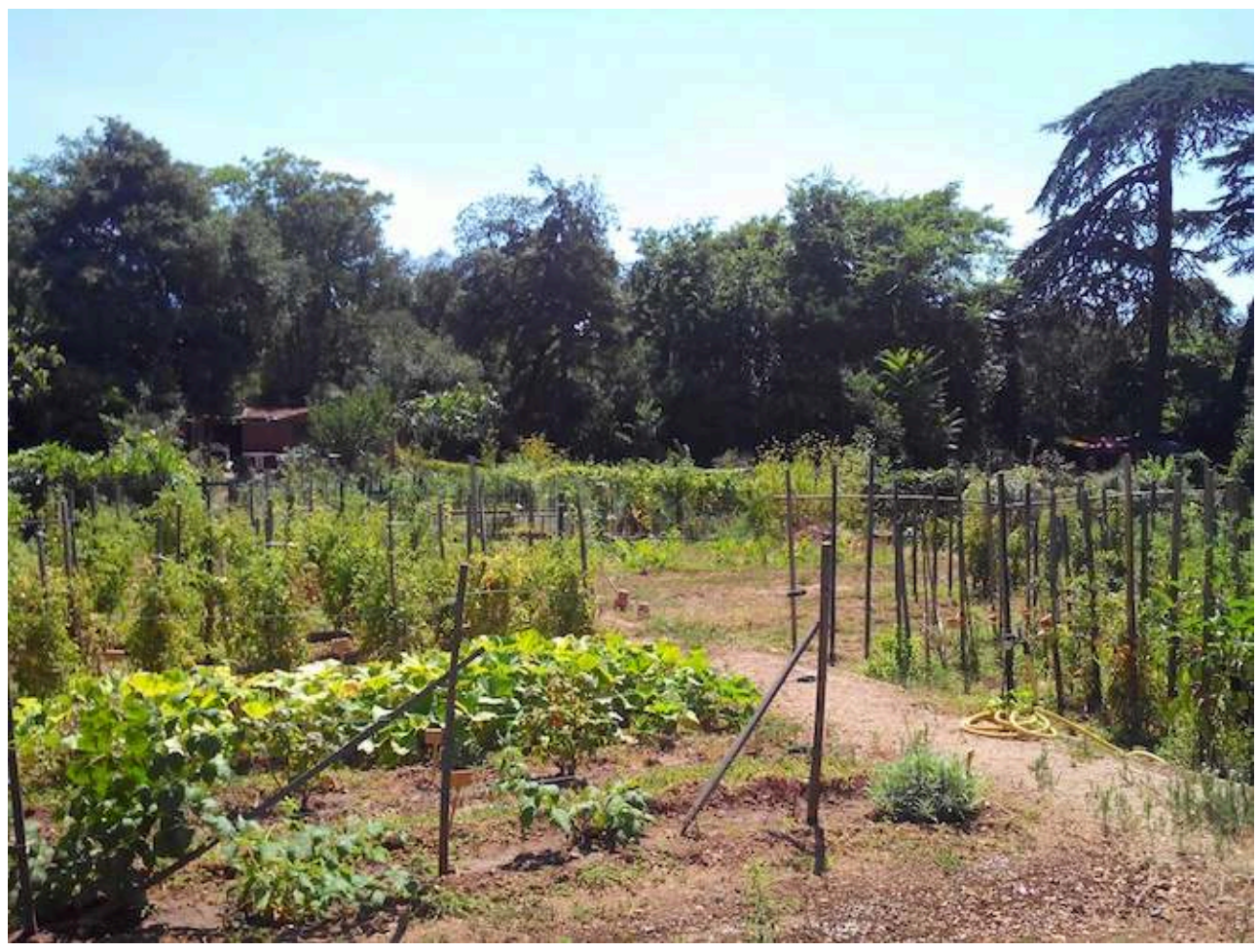

De ce conseil, le comité local de Marseille de la FNJFC fait une stratégie. Depuis que le projet du boulevard urbain sud (BUS) se précise (il entre en phase d'enquête publique en octobre 2015) et menace d'amputer $30 \%$ de son site historique de Mazargues, il déploie une véritable politique de défense au sein de laquelle la biodiversité tient une place centrale. Il s'agit ainsi d'évaluer la valeur écologique du jardin et de mesurer la force de cet argument. À cet effet, le bureau ouvre le site à la recherche. Un biologiste de l'université d'Aix-Marseille entame un inventaire de la végétation et un suivi floristique. La Ligue de protection des oiseaux (LPO) évalue la richesse ornithologique. 
La biodiversité espérée par l'association relève alors de l'extraordinaire : il suffirait que les jardins familiaux abritent une espèce rare ou protégée pour que le tracé du BUS soit totalement remis en cause. Mais, les résultats démontrent que la biodiversité en présence, bien que très abondante, ne relève que de l'ordinaire. L'association prend acte de ce résultat et modifie sa stratégie. Il s'agit désormais de se battre pour une biodiversité, certes ordinaire, mais exceptionnelle par son épaisseur historique (les jardins et certains arbres sont plus que centenaires) et par sa singularité dans un tel contexte urbain (le site occupe 4 hectares dans un quartier très densément peuplé). La biodiversité se mue donc en patrimoine. Elle ne fige pas pour autant le jardin. Au contraire, elle donne lieu à de microprojets : des ruches, des nichoirs et des hôtels à insectes sont installés; un chercheur de l'université d'Aix-Marseille y établit une importante collection de plantes méditerranéennes ; des étudiants en paysage étudient l'intégration du site dans la trame verte urbaine marseillaise; le jardin est investigué par l'ANR JASSUR.

\section{Conclusion}

La confrontation des deux échelles d'analyse mobilisées dans le présent article montre donc que les jardins familiaux sont désormais autant au service de la biodiversité que celle-ci est à leur service. En effet, à l'échelle nationale, la biodiversité participe de la stratégie de réaffirmation de la Fédération nationale des jardins familiaux et collectifs. Dans un climat de plus en plus concurrentiel, il s'agit, pour elle, de démontrer que les potagers urbains sont des lieux de nature à part entière qui permettent le maintien et le développement de la faune et de la flore et qui fournissent, de ce fait, des services écosystémiques variés aux territoires. Ce faisant, elle soutient, depuis le début des années 2000, un processus d'évolution engageant autant les discours qu'elle défend que les pratiques qu'elle promeut à l'égard d'une biodiversité de mieux en mieux comprise et utilisée. Dans ce contexte, la biodiversité fait office de véritable programme d'actions, induisant un positionnement et des modes de faire renouvelés pour l'ensemble des acteurs fédératifs au premier rang desquels figure le bureau d'étude Sites et Jardins. De plus en plus organisée autour de savoirs et savoir-faire environnementalistes, cette structure porte des projets dont la durabilité repose en grande partie sur la prise en compte, dans l'aménagement, de la faune et de la flore. Cependant, malgré ces rapports symbiotiques grandissants, les relations qui se tissent entre la biodiversité et les jardins familiaux restent encore régies, à l'échelle locale, par des logiques ambivalentes. Car, d'un côté, les enquêtes réalisées sur Marseille montrent que la biodiversité est désormais assez maîtrisée par les associations de jardiniers pour être mobilisée comme un argument majeur dans la protection des jardins familiaux face à l'urbanisation. Cette mobilisation les conduit à dépasser des conceptions uniquement centrées sur des espèces extraordinaires (rares ou protégées) pour intégrer, à leurs stratégies de défense, des réflexions sur des espèces certes plus ordinaires mais très abondantes dans les jardins familiaux. Il s'agit alors pour ces associations de présenter leurs potagers à la fois comme des réserves de biodiversité ordinaire, rendues exceptionnelles par les surfaces qu'elles occupent au sein du tissu urbain, et comme des patrimoines verts vivants, eu égard à l'épaisseur historique des sites cultivés. Dès lors, la biodiversité ordinaire que renferment les jardins familiaux et qu'entretiennent leurs jardiniers est désignée comme un bien commun du territoire urbain dont la préservation engage, au-delà de la petite communauté des cultivateurs, 
l'ensemble des habitants de la ville. Mais, d'un autre côté, les enquêtes réalisées montrent aussi que les pratiques quotidiennes des jardiniers restent fortement marquées par des considérations différenciées à l'égard de cette biodiversité ordinaire. Si leur intérêt pour la flore cultivée ne cesse de croître et de s'orienter vers des gammes de plus en plus larges de variétés, la flore spontanée demeure inconsidérée. Par ailleurs, si la faune sauvage visible est estimée en tant qu'incarnation de l'idée de nature au jardin (les oiseaux, les insectes et certains animaux sont appréciés comme de véritables auxiliaires), la faune des sols, abondante mais invisible, est largement ignorée.

Face à ces constats, deux pistes d'ouverture s'offrent à nous. D'ordre scientifique, la première appelle à accroître les efforts de recherche sur les sols et leur biodiversité. Malgré l'intérêt grandissant qu'ils suscitent, ces derniers restent une sorte d'angle mort, d'impensé, de l'aménagement urbain, en général, et de l'écosystème des jardins, en particulier. Il appartient, sans doute, aux scientifiques de non seulement étendre les connaissances à leur sujet mais encore de trouver des modes opératoires de transmission et de conscientisation efficaces. D'ordre projectuel, la seconde conduit à considérer les limites de l'entrée sur les jardins familiaux par les seuls services ou fonctions. Bien qu'essentielle pour comprendre la portée sociale, culturelle, économique ou environnementale de ces potagers, elle ne saurait suffire pour penser leur conception. Car, au-delà de leur dimension utilitaire (Maris, 2014), les notions de services et de fonctions réduisent les jardins familiaux au rang de simples supports ou de simples outils. Elles appellent à des aménagements bien moins fondés sur la créativité et la fabrique de lieux uniques que sur des méthodologies et des techniques éprouvées, reproductibles, destinées à faire émerger des effets attendus. Ce faisant, elles placent le contenu au-dessus du contenant et privilégient le potager, cantonné à sa dimension productive, au détriment du jardin dont la portée ontologique (Assunto, 1973) ne peut être appréhendée par la seule notion de service. Ici, c'est la forme qui apparaît comme l'angle mort, l'impensé, du projet. Véritables leitmotivs des maîtrises d'ouvrage, les services et les fonctions, posés à la fois comme moyens et finalités de l'action, contraignent bien souvent les maîtres d'œuvre à des réponses d'autant plus convenues qu'elles s'appuient sur des budgets dérisoires. Or, en l'absence de qualité urbaine et paysagère, les services et les fonctions, si importants soient-ils, ne peuvent justifier, à eux seules, l'éclosion du bien commun au cœur des potagers. Dès lors, il appartient aux concepteurs de métier, au premier rang desquels figurent les paysagistes, d'investir plus massivement ce champ et de transformer le renouveau des jardins familiaux en véritable projet de paysage.

Les auteurs tiennent ici à remercier de leur précieuse collaboration Gil Melin, Hervé Bonnavaud, Jérôme Clément, Florent Roubinet, Honoré et Nicole Gelli ainsi que l'ensemble de la FNJFC. Ils tiennent également à témoigner de leur gratitude à l'égard de l'ANR Villes et Bâtiments durables pour son aide dans le cadre du programme JASSUR. 


\section{BIBLIOGRAPHIE}

Assunto, R., Il paesaggio e l'estetica, Naples, Giannini, 1973, 2 vol.

Aronson, M.F.J., Handel, S.N., Clemants, S.E., « Fruit type, life form and origin determine the success of woody plant invaders in an urban landscape », Biological Invasions, $n^{\circ}$ 9, 2007, p. $465-475$.

Aubry, C., « Les agricultures urbaines et les questionnements de la recherche », Pour. Agricultures Urbaines, $n^{\circ} 224$, Paris, GREP, 2014, p. 35-49.

Berendse, F., « Effects of Dominant Plant Species on Soils during Succession in Nutrient-poor Ecosystems ", Biogeochemistry, $\mathrm{n}^{\circ}$ 42, 1998, p. 73-88.

Bonnal, P., Bonin, M., Aznar, O., « Les évolutions inversées de la multifonctionnalité de l'agriculture et des services environnementaux ", Vertigo. La revue électronique en sciences de l'environnement, vol. 12, nº 3, Montréal, 2012, URL : https://vertigo.revues.org/12882.

Cabedoce, C., Pierson, P., Cent ans d'histoire des jardins ouvriers : 1896-1996. La Ligue française du Coin de Terre et du Foyer, Grâne, Éditions Creaphis, 1996.

Chenot, E.-D., Douay, F., Dumat, C., Pernin, C., Pourrut, B., Schwartz, C. (dir.), Jardins potagers : terres inconnues? Les Ulis, EDP Sciences, 2012, 171 p.

Consalès, J.-N., « Les jardins familiaux marseillais : laboratoires territoriaux d'une agriculture urbaine en Méditerranée », Méditerranée, $n^{\circ} 95$ (3-4), 2000, p. 81-88.

Consales, J.-N., « Jardins familiaux et développement durable : entre discours théoriques et actes concrets ", dans Da Lage, A., Amat, J.-P., Frérot, A.-M., Guichard-Anguis, S., Julien-Laferrière, B., Wicherek, S.-P., (dir..), L’Après développement durable, Paris, Ellipses, 2008, p. 203-211.

Corajoud, M., « Le projet de paysage, lettre aux étudiants », dans Le Jardinier, l'Artiste, l'Ingénieur, Besançon/Paris, Les Éditions de l'imprimeur, 2000, p. 37-50.

Cortet, J., Vauflery, A. G.-D., Poinsot-Balaguer, N., Gomot, L., Texier, C., Cluzeau, D., « The use of invertebrate soil fauna in monitoring pollutant effects », European Journal of Soil Biology, ${ }^{\circ} 35$, 1999 , p. 115-134.

Díaz, S., Cabido, M., « Vive la différence : plant functional diversity matters to ecosystem processes », Trends in Ecology \& Evolution, $\mathrm{n}^{\circ}$ 16, 2001, p. 646-655.

Dubost, F., Jardins ordinaires, Paris, L'Harmattan, 2000.

Duchemin, E., Wegmuller, F., Legault, M., « Agriculture urbaine : un outil multidimensionnel pour le développement des quartiers ", Vertigo. La revue électronique en sciences de l'environnement, vol. 10, n 2, Montréal, 2010, http://vertigo.revues.org/10436.

Fleury, A., Donadieu, P., « De l'agriculture périurbaine à l'agriculture urbaine », Courrier de l'Environnement de l'Inra, $\mathrm{n}^{\circ}$ 31, Paris, 1997, p. 45-61.

Frauenfelder, A., Delay, C., Scalambrin, L., « Potagers urbains vs jardins familiaux ? Réforme urbaine et controverses autour du beau jardin et son usage légitime », Espaces et Sociétés, $\mathrm{n}^{\circ} 158$, Paris, Éditions Erès, 2014, p. 67-81.

Guyon, F., " Les jardins familiaux aujourd'hui : des espaces socialement modulés ", Espaces et Sociétés, nº 134, Paris, Éditions Erès, 2008, p. 131-147. 
Hooper, D. U., Chapin, F. S., Ewel, J.-J., Hector, A., Inchausti, P., Lavorel, S., Lawton, J. H., Lodge, D. M., Loreau, M., Naeem, S., Schmid, B., Setälä, H., Symstad, A. J., Vandermeer, J., Wardle, D. A., «Effects of biodiversity on ecosystem functioning : a consensus of current knowledge », Ecological Monographs, $\mathrm{n}^{\circ} 75,2005$, p. 3-35.

Joimel, S., Schwartz, C., Auclerc, A., Bel, J., Branchu, P., Chenot, E-D., Consalès, J.N., Hedde, M., Magnus, B., Morel, J.-L., Cortet, J., « Soil invertebrates as bioindicators of soil quality in urban vegetable gardens ", The First Global Soil Biodiversity Conference, Dijon, Cenre des congrès, 2 au 5 décembre 2014.

Joimel, S., «Biodiversité et caractéristiques physicochimiques des sols de jardins associatifs urbains français ", thèse de doctorat en sciences agronomiques, université de Lorraine, Vandœuvre-lès-Nancy, 2015.

Kuznetsova, N. A., « New Approaches to the Assessment of Structural Organization of Communities in Springtails (Hexapoda : Collembola) », Russian Journal of Ecology, $n^{\circ} 34,2003$, p. 248-254.

Kleyer, M., « Distribution of plant functional types along gradients of disturbance intensity and resource supply in an agricultural landscape ", Journal of Vegetation Science, n 10, 1999, p. 697-708.

Knapp, S., Dinsmore, L., Fissore, C., Hobbie, S. E., Jakobsdottir, I., Kattge, J., King, J.-Y., Klotz, S., McFadden, J.-P., Cavender-Bares, J., « Phylogenetic and functional characteristics of household yard floras and their changes along an urbanization gradient ", Ecology, $n^{\circ} 93,2012$, p. 83-98.

Lovell, S. T., « Multifunctional Urban Agriculture for Sustainable Land Use Planning in the United States », Sustainability, $\mathrm{n}^{\circ}$ 2, 2010, p. 2 499- 2522.

Maris, V., Nature à vendre. Les limites des services écosystémiques, Versailles, éditions Quæ, 2014.

Millennium Ecosystem Assessment, Ecosystems and Human Well-being 5, Washington, DC, Island Press, 2005.

Mougenot, C., Prendre soin de la nature ordinaire, Versailles, éditions Quæ, 2003.

Pashchenko, O., Consalès, J. N., « Les jardins collectifs : derrière une seule notion, des réalités territoriales contrastées », Durabilis, magazine expérientiel de l'environnement et du développement durable, Montpellier, 2010.

Potapow, M, « Synopses on palaearctic collembola Isotomidae », Staatliches Museum für Naturkunde, Görlitz, Germany Staatliches Museum für Naturkunde, 2011.

Pourias, J., Daniel, A.-C., Aubry, C., « La fonction alimentaire des jardins associatifs urbains en question », Pour, $\mathrm{n}^{\circ}$ 215-216, Paris, GREP, 2014, p. 333-347

Santorufo, L., Van Gestel, C. A. M., Rocco, A., Maisto, G., « Soil invertebrates as bioindicators of urban soil quality », Environmental Pollution, $n^{\circ} 161,2012$, p. 57-63.

Santorufo, L., Cortet, J., Arena, C., Goudon, R., Rakoto, A., Morel, J.-L., Maisto, G., « An assessment of the influence of the urban environment on collembolan communities in soils using taxonomyand trait-based approaches ", Applied Soil Ecology, $\mathrm{n}^{\circ} 78,2014$, p. 48-56.

Scheromm, P., « Les jardins collectifs, entre nature et agriculture », Métropolitiques, 2013, URL : www.metropolitiques.eu/Les-jardins-collectifs-entre.html.

Schwartz, C., « Facteurs de qualité des sols de jardins de l'est-mosellan », mémoire de DEA sciences agronomiques, Institut national polytechnique de Lorraine, École nationale supérieure d'agronomie et des industries alimentaires, 1993, $44 \mathrm{p}$. 
Smith, R. M., Thompson, K., Hodgson, J. G., Warren, P. H., Gaston, K. J., « Urban domestic gardens (IX) : Composition and richness of the vascular plant flora, and implications for native biodiversity ", Biological Conservation, $\mathrm{n}^{\circ} 129,2006$, p. 312-322.

Weber, F., L'Honneur des jardiniers. Les potagers dans la France du XXe siècle, Paris, Belin, 2000.

Wegmuller, F., Duchemin, E., « Multifonctionnalité de l'agriculture urbaine à Montréal : étude des discours au sein du programme des jardins communautaires », Vertigo. La revue électronique en sciences de l'environnement, vol. 10, $\mathrm{n}^{\circ}$ 2, Montréal, 2010, URL : http://vertigo.revues.org/10445.

\section{NOTES}

1. L'article L561-1 de la proposition de loi de 2001 range sous le terme générique de jardins collectifs :

- les jardins familiaux, définis comme «les terrains divisés en parcelles, affectés par les collectivités territoriales ou par les associations de jardins familiaux à des particuliers y pratiquant le jardinage pour leurs propres besoins et ceux de leur famille, à l'exclusion de tout usage commercial. [...]»;

- les jardins d'insertion définis comme "les jardins créés ou utilisés en vue de favoriser la réintégration des personnes en situation d'exclusion ou en difficulté sociale ou professionnelle $[\ldots] »$;

- les jardins partagés, définis comme «les jardins créés ou animés collectivement, ayant pour objet de développer des liens sociaux de proximité par le biais d'activités sociales, culturelles ou éducatives et étant accessibles au public.»

2. Nous tenons, à cet égard, à encore vivement remercier la FNJFC et plus particulièrement Hervé Bonnavaud de nous avoir permis d'accéder à l'intégralité des magazines disponibles sous format numérique.

3. 10 années valent, en fait, 60 volumes. Mais il faut soustraire, à notre corpus, un volume non encore publié durant l'année en cours et 3 volumes non disponibles sous format numérique ( $n^{\circ} 469, n^{\circ} 470, n^{\circ} 472$ de l'année 2012).

4. Tropes est un logiciel développé par Pierre Molette et Agnès Landré sur la base des travaux de Rodolphe Ghiglione (URL : http://www.tropes.fr/)

5. Mots indicateurs interrogés : adventices, bio, biodiversité, développement durable, écologie, écosystème, environnement, faune, flore, Grenelle de l'environnement, mauvaises herbes, nature, nuisible, pesticide, pollution, trame verte et bleue, urbanisation, végétation.

6. Les quatre phases sont : « une phase informative auprès des élus et des techniciens; une phase de concertation avec les acteurs de la ville et les habitants pour élaborer un cahier des charges; une phase d'élaboration et de présentation du projet avec la remise des plans d'aménagement du site ; une phase technique : le bureau d'études est en charge du suivi des travaux et assure les réunions de chantier jusqu'à la livraison. " (URL: http://www.jardins-familiaux.asso.fr/ conception.html)

7. Ce travail de sélection s'est fait dans le cadre de la thèse de doctorat de Sophie Joimel (2015). Il s'est fondé sur une classification de jardins familiaux situés dans trois agglomérations (Marseille, Grand Nancy et Nantes) aux climats différents (méditerranéen, semi-continental, océanique) et placés dans des contextes urbains variés (habitation, industriel, mixte). Au total, 104 parcelles situées sur 26 sites ont été investiguées, dont 36 à Marseille (sur 10 sites différents).

8. La parcelle de jardin est quadrillée avec des carrés d'un mètre de côté pour la zone cultivée et de trente centimètres de côté pour la zone non cultivée. Les microarthropodes sont prélevés au centre de douze de ces quadrats sélectionnés aléatoirement. 


\section{RÉSUMÉS}

Le présent article étudie les relations qui se tissent entre les jardins familiaux et la notion de biodiversité. Pour ce faire, il confronte deux échelles d'analyse. À l'échelle nationale, il montre que la biodiversité procède pleinement de la stratégie de la Fédération nationale des jardins familiaux et collectifs en faveur de la (ré)affirmation de ces potagers urbains. Cette structure accompagne ainsi des projets pensés comme durables et conçus pour être favorables à la faune et à la flore. À l'échelle locale, les relevés écologiques réalisés dans des sites marseillais désignent les jardins familiaux comme des réservoirs d'une biodiversité abondante mais ordinaire. Face à l'urbanisation, celle-ci sert souvent d'argument de défense pour les associations de gestion. Les jardins familiaux génèrent, par ailleurs, des considérations et des pratiques renouvelées, mais encore fortement empreintes de représentations et d'ambiguïtés à l'égard de la notion de biodiversité. De fait, la flore spontanée et la faune du sol demeurent des impensés de l'écosystème du potager.

This article studies the relationships between allotment gardens and the notion of biodiversity. To do so, it compares two levels of analysis. On the national level, it shows that biodiversity is an integral part of the strategy developed by the National Federation of Allotment and Community Gardens (in French Fédération nationale des jardins familiaux et collectifs) in support of these urban vegetable gardens. This association facilitates projects designed to be sustainable and to provide a favourable environment for plant and animal life. At the local level, ecological surveys conducted in certain locations in Marseilles have designated allotment gardens as the reservoirs of an abundant although ordinary biodiversity. In a context of urbanisation, this often serves as an argument in favour of community garden management associations. Allotment gardens also give rise to renewed considerations and practises, but ones which are greatly marked by representations and ambiguities regarding the notion of biodiversity. As a result, the spontaneous growth of plants and animal life in the soil are the unplanned results of the vegetable garden.

\section{INDEX}

Mots-clés : jardins collectifs, jardins familiaux, biodiversité, approche projectuelle, approche écologique

Keywords : community gardens, allotment gardens, biodiversity, project approach, ecological approach

\section{AUTEURS}

\section{JEAN NOËL CONSALÈS}

Urbaniste et géographe, Jean Noël Consalès est maître de conférences à l'Institut d'urbanisme et d'aménagement régional, Aix-Marseille université, UMR 7303, TELEMME, CNRS.

jean-noel.consales[at]univ-amu[dot]fr 


\section{SOPHIE JOIMEL}

Écologue du sol, Sophie Joimel est maître de conférences contractuel à AgroParistech, UMR INRA ECOSYS.

soboulanger[at]grignon.inra[dot]fr

\section{FRANCE CORDIER}

France Cordier est urbaniste, Institut d'urbanisme et d'aménagement régional, Aix-Marseille université, UMR 7303, TELEMME, CNRS.

france.cordier[at]gmail[dot]com

\section{CLOÉ JARENO}

Ingénieur paysagiste, Cloé Jareno est doctorante CIFRE, Aix-Marseille université, EA 889-LIEU, Scop SaluTerre.

chloe.jareno[at]saluterre[dot]com

\section{ÉLODIE-DENISE CHENOT}

Chimiste, Élodie-Denise Chenot est ingénieur d'études en environnement au laboratoire Sols et Environnement, UMR 1120, université de Lorraine-INRA.

elodie.chenot[at]univ-lorraine[dot]fr

\section{CHRISTOPHE SCHWARTZ}

Agronome et pédologue urbain, Christophe Schwartz est professeur en pédologie urbaine à l'université de Lorraine, UMR 1120, laboratoire Sols et Environnement, Vandœuvre-lès-Nancy, INRA, UMR 1120.

christophe.schwartz[at]univ-lorraine[dot]fr 